Article

\title{
Integrated MicroRNA-mRNA Profiling Identifies Oncostatin $M$ as a Marker of Mesenchymal-Like ER-Negative/HER2-Negative Breast Cancer
}

\author{
Giulia Bottai ${ }^{1}$, Lixia Diao ${ }^{2}$, Keith A. Baggerly ${ }^{2}$, Laura Paladini ${ }^{1}$, Balázs Győrffy ${ }^{3,4}$, \\ Carlotta Raschioni ${ }^{1}$, Lajos Pusztai ${ }^{5}$, George A. Calin ${ }^{6,7}$ and Libero Santarpia ${ }^{1, *}$
}

1 Oncology Experimental Therapeutics, Humanitas Clinical and Research Institute, 20089 Rozzano-Milan, Italy; giulia.bottai@humanitasresearch.it (G.B.); laura.paladini@humanitasresearch.it (L.P.);

carlotta.raschioni@humanitas.it (C.R.)

2 Department of Bioinformatics and Computational Biology, The University of Texas MD Anderson Cancer Center, Houston, TX 77030, USA; ldiao@mdanderson.org (L.D.); kabagger@mdanderson.org (K.A.B.)

3 2nd Department of Pediatrics, Semmelweis University, H-1094 Budapest, Hungary

4 MTA TTK Lendület Cancer Biomarker Research Group, Hungarian Academy of Sciences, H-1117 Budapest, Hungary; gyorffy.balazs@ttk.mta.hu

5 Department of Breast Medical Oncology, Yale University, Yale Cancer Center, New Haven, CT 06520, USA; lajos.pusztai@yale.edu

6 Department of Experimental Therapeutics, The University of Texas MD Anderson Cancer Center, Houston, TX 77030, USA; gcalin@mdanderson.org

7 RNA Interference and Non-Coding RNA Center, The University of Texas MD Anderson Cancer Center, Houston, TX 77030, USA

* Correspondence: libero.santarpia@humanitasresearch.it or liberosantarpia@yahoo.it; Tel.: +39-02-8224-5173; Fax: +39-02-8224-5191

Academic Editor: Nicoletta Sacchi

Received: 8 December 2016; Accepted: 13 January 2017; Published: 19 January 2017

\begin{abstract}
MicroRNAs (miRNAs) simultaneously modulate different oncogenic networks, establishing a dynamic system of gene expression and pathway regulation. In this study, we analyzed global miRNA and messenger RNA (mRNA) expression profiles of 17 cell lines representing different molecular breast cancer subtypes. Spearman's rank correlation test was used to evaluate the correlation between miRNA and mRNA expression. Hierarchical clustering and pathway analysis were also performed. Publicly available gene expression profiles $(n=699)$ and tumor tissues $(n=80)$ were analyzed to assess the relevance of key miRNA-regulated pathways in human breast cancer. We identified 39 significantly deregulated miRNAs, and the integration between miRNA and mRNA data revealed the importance of immune-related pathways, particularly the Oncostatin M (OSM) signaling, associated with mesenchymal-like breast cancer cells. OSM levels correlated with genes involved in the inflammatory response, epithelial-to-mesenchymal transition (EMT), and epidermal growth factor (EGF) signaling in human estrogen receptor (ER)-negative/human epidermal growth factor receptor 2 (HER2)-negative breast cancer. Our results suggest that the deregulation of specific miRNAs may cooperatively impair immune and EMT pathways. The identification of the OSM inflammatory pathway as an important mediator of EMT in triple-negative breast cancer (TNBC) may provide a novel potential opportunity to improve therapeutic strategies.
\end{abstract}

Keywords: breast cancer; microRNAs; molecular subtypes; immune response; Oncostatin M

\section{Introduction}

Breast cancer is a major cause of cancer death in women worldwide. Recent advancements in the understanding of cancer biology and oncogenic mechanisms have led to the refinement of breast 
cancer classification, highlighting the molecular and clinical heterogeneity of this disease. Indeed, distinct signaling pathways, genetic and epigenetic aberrations, and gene expression profiles have been associated with different breast cancer subtypes [1-7]. In particular, triple-negative breast cancer (TNBC), which lacks the expression of estrogen receptor (ER), progesterone receptor (PR), and human epidermal growth factor receptor 2 (HER2), encompasses a heterogeneous collection of tumors $[8,9]$. Specific TNBC subgroups are enriched for genes involved in the epithelial-to-mesenchymal transition (EMT) process, which has been associated with cancer progression, poor prognosis, and resistance to therapy [9]. Furthermore, cancer-related inflammation is now recognized as a hallmark of cancer, and anti-tumor immune responses are emerging as important predictors of outcome and response to therapies in TNBC $[10,11]$. Several molecular messengers, such as chemokines and cytokines, are able to modulate the reciprocal interaction between breast cancer cells and the immune microenvironment, thus supporting tumor development and progression [10]. Considering the complexity and heterogeneity of breast cancer, the identification of reliable subtype-specific molecules, which function to simultaneously modulate different oncogenic networks, may provide novel insights into the dynamic architecture of cancer-related pathways and an opportunity to optimize therapeutic strategies.

MicroRNAs (miRNAs) are recognized as master modulators of multiple biological and pathological processes, establishing a complex combinatorial system of gene expression and pathway regulation, as well as new therapeutic targets in different cancers $[12,13]$. Furthermore, recent studies highlighted the relevance of miRNAs in regulating the crosstalk between cancer and immune cells in the tumor microenvironment $[14,15]$. Deregulation of miRNAs expression through genetic and epigenetic alterations, impairment of the biogenesis machinery, and alterations of the tumor microenvironment (e.g., imbalance of immune cells and related cytokines), are frequently observed in human cancers, including breast cancer [12-15].

In this study, we integrated miRNA-messenger RNA (mRNA) expression profiles to identify specific pathway perturbations that characterize triple-negative/mesenchymal-like breast cancer cell lines and human breast tumors.

\section{Results}

\subsection{Specific miRNA Expression Patterns Define Molecularly Different Human Breast Cancer Cell Lines}

We analyzed the genome-wide miRNA profiles of 17 cell lines representative of different breast cancer molecular subtypes (Table S1) [16]. By selecting only miRNAs expressed in at least nine cell lines, we identified 39 miRNAs significantly deregulated between triple-negative/mesenchymal-like and non-mesenchymal-like (luminal/HER2-positive) cells (Benjamini-Hochberg-adjusted $p \leq 0.05$ ). Of these, 22 and 17 miRNAs were up-regulated and down-regulated in the triple-negative/mesenchymal-like cells, respectively (Table 1).

Hierarchical clustering analysis revealed that the 17 breast cancer cell lines were separated into two main branches based on differences in the expression of the 39 miRNAs (Figure 1). Interestingly, the two HER2-positive cell lines (AU565 and SKBR3) formed a separate sub-cluster of the non-mesenchymal-like branch, indicating the robustness of our classification (Figure 1). These results indicate that miRNA expression profiles are able to characterize molecularly different breast cancer cell lines, and confirm that intrinsic features of breast cancer subtypes are evident also at the level of miRNAs expression. 
Table 1. The 39 significantly deregulated microRNAs (miRNAs) in triple-negative/mesenchymal-like breast cancer cell lines.

\begin{tabular}{|c|c|c|c|}
\hline \multicolumn{2}{|c|}{ Up-Regulated } & \multicolumn{2}{|c|}{ Down-Regulated } \\
\hline miRNA & $p$-Value ${ }^{1}$ & miRNA & $p$-Value ${ }^{1}$ \\
\hline miR-29a & $1.52 \times 10^{-2}$ & miR-34a & $5.43 \times 10^{-4}$ \\
\hline miR-31 & $6.26 \times 10^{-3}$ & miR-141 & $3.08 \times 10^{-8}$ \\
\hline miR-100 & $8.54 \times 10^{-3}$ & miR-148a & $5.49 \times 10^{-4}$ \\
\hline $\operatorname{miR}-125 b$ & $1.98 \times 10^{-2}$ & $\operatorname{miR}-190 \mathrm{~b}$ & $8.54 \times 10^{-3}$ \\
\hline miR-130a & $1.90 \times 10^{-2}$ & miR-193a-3p & $6.26 \times 10^{-3}$ \\
\hline miR-138 & $4.41 \times 10^{-3}$ & miR-196a & $3.08 \times 10^{-2}$ \\
\hline miR-140-3p & $1.06 \times 10^{-2}$ & miR-200a & $6.32 \times 10^{-7}$ \\
\hline miR-143 & $1.06 \times 10^{-2}$ & miR-200b & $3.08 \times 10^{-8}$ \\
\hline miR-145 & $2.69 \times 10^{-2}$ & miR-200c & $2.85 \times 10^{-7}$ \\
\hline miR-146a & $4.01 \times 10^{-3}$ & miR-203 & $1.81 \times 10^{-2}$ \\
\hline miR-146b-5p & $6.24 \times 10^{-3}$ & miR-205 & $2.69 \times 10^{-2}$ \\
\hline miR-155 & $3.86 \times 10^{-3}$ & miR-301a & $7.13 \times 10^{-3}$ \\
\hline miR-199a-3p/199b-3p & $9.11 \times 10^{-3}$ & miR-335 & $1.05 \times 10^{-2}$ \\
\hline miR-199a-5p & $2.18 \times 10^{-3}$ & miR-363 & $8.86 \times 10^{-3}$ \\
\hline miR-221 & $1.88 \times 10^{-3}$ & miR-375 & $1.49 \times 10^{-2}$ \\
\hline miR-222 & $2.11 \times 10^{-3}$ & miR-429 & $1.06 \times 10^{-2}$ \\
\hline $\operatorname{miR}-376 c$ & $3.66 \times 10^{-5}$ & miR-934 & $3.08 \times 10^{-2}$ \\
\hline $\operatorname{miR}-455-3 p$ & $1.81 \times 10^{-2}$ & & \\
\hline miR-582-5p & $3.64 \times 10^{-3}$ & & \\
\hline miR-886-3p & $6.24 \times 10^{-3}$ & & \\
\hline miR-886-5p & $6.26 \times 10^{-3}$ & & \\
\hline miR-1290 & $4.41 \times 10^{-3}$ & & \\
\hline
\end{tabular}

${ }^{1}$ Benjamini-Hochberg adjusted $p$-value.

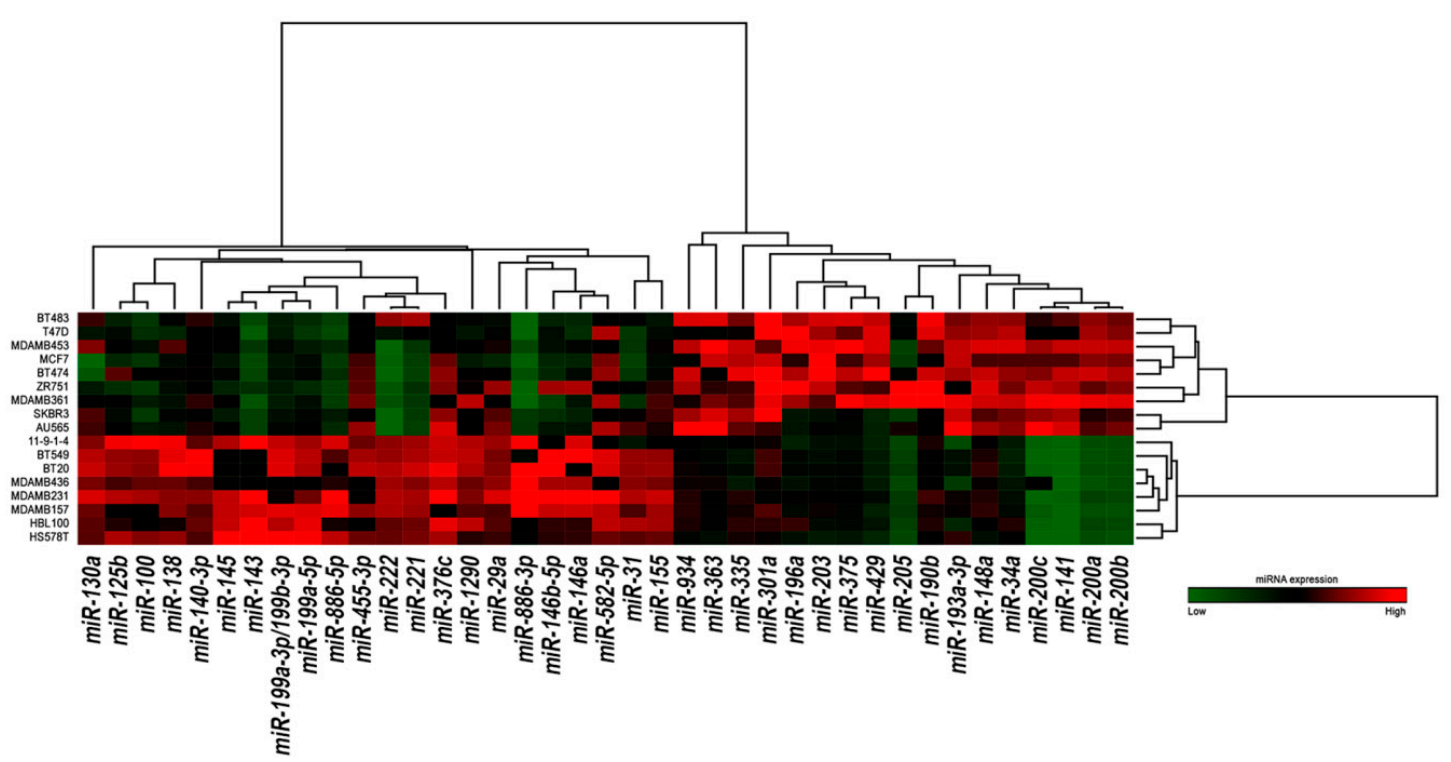

Figure 1. Hierarchical clustering of the 39 most differentially expressed miRNAs in 17 breast cancer cell lines. Expression values of miRNAs are represented in a matrix format, with columns indicating miRNAs and rows indicating samples. High expression values are color-coded red, and low expression values are color-coded green. Hierarchical clustering of both miRNAs (columns) and samples (rows) are shown. 


\subsection{Correlation between miRNA and $m R N A$ Expression Profiles and Pathway Analysis}

To investigate the role of miRNAs in the regulation of multiple transcriptomic networks, we assessed the correlation between the expression of the 39 selected miRNAs and the genome-wide mRNA profiles of the 17 breast cancer cell lines. Collectively, we found 4813 genes significantly correlated with the 39 deregulated miRNAs. Among these genes, we selected the mRNAs with the highest absolute correlation values for each miRNA using a false discovery rate (FDR) threshold of 0.05 and performed a clustering analysis of miRNA-mRNA integrated profiles (Figure 2, Table S2). The integration of expression data allowed for an accurate clustering of breast cancer cells into mesenchymal-like and non-mesenchymal-like subtypes, with the exception of BT20 cell line (Figure 2). This misclassification could be explained by the unclassified morphology of this TNBC cell line, as reported by the American Type Culture Collection (ATCC; Table S1).

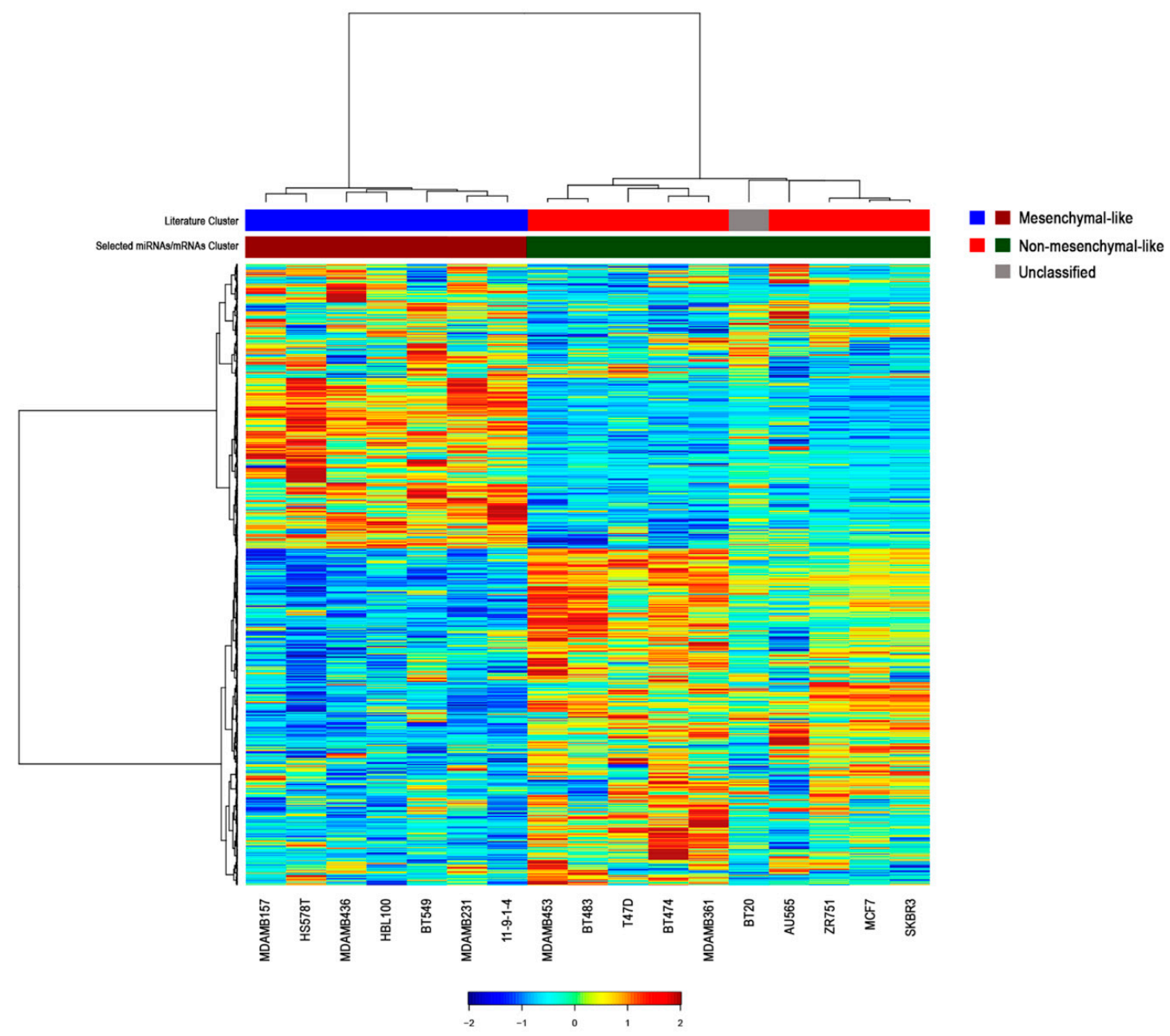

Figure 2. Hierarchical clustering of the most significant messenger RNAs (mRNAs) correlated with the 39 miRNAs in breast cancer cell lines. To generate the integrated profiles, we selected the mRNAs with the highest absolute correlation values for each miRNA among the 4813 genes significantly correlated with the 39 miRNAs. The color in the bars beneath the clustering illustrates the two different molecular subgroups, triple-negative/mesenchymal-like and non-mesenchymal-like (luminal/HER2-positive) subtypes. Literature cluster was assembled according to American Type Culture Collection (ATCC) and Neve et al. [16]. The unclassified BT20 cell line is depicted in grey. Hierarchical clustering of both mRNAs (rows) and samples (columns) are shown. 
Predicted and validated targets of the 39 significantly deregulated miRNAs were identified using miRBase, TargetScan, and Tarbase tools. Only a few miRNAs showed an overlap between the correlated mRNAs and the predicted targets (Table S3). Even though concordant genes may be considered as direct miRNA targets, the reduced number of overlapping genes indicates that indirect modulation mechanisms may be prevalent on direct target suppression, further strengthening the complexity and the extensive regulatory role of miRNAs on gene expression in breast cancer.

Subsequently, we performed a pathway analysis to gain further insight into the biological functions of these miRNAs in breast cancer. The association of correlated miRNAs-mRNAs with related activated pathways is displayed in a heatmap with diverse colors corresponding to different levels of significance (Figure 3). The most significant association (FDR $\leq 1.00 \times 10^{-4}$ ) was found between two miRNAs that were up-regulated in triple-negative/mesenchymal-like cells, miR-146b-5p and miR-155, and the pathway of Oncostatin M (OSM), which is a member of the multifunctional interleukin 6 (IL-6) family of cytokines (Figure 3). The OSM pathway was also consistently associated with the expression of miR-31 (FDR $\leq 5.00 \times 10^{-4}$ ), and miR-29a, miR-199a-3p/199b-3p, miR-199a-5p, miR-200a, miR-221, and miR-222 (FDR level $\leq 5.00 \times 10^{-3}$ ) (Table 2). Other immune-related and oncogenic pathways were found to significantly correlate with specific miRNA-mRNA networks at different FDR levels (Table 2).

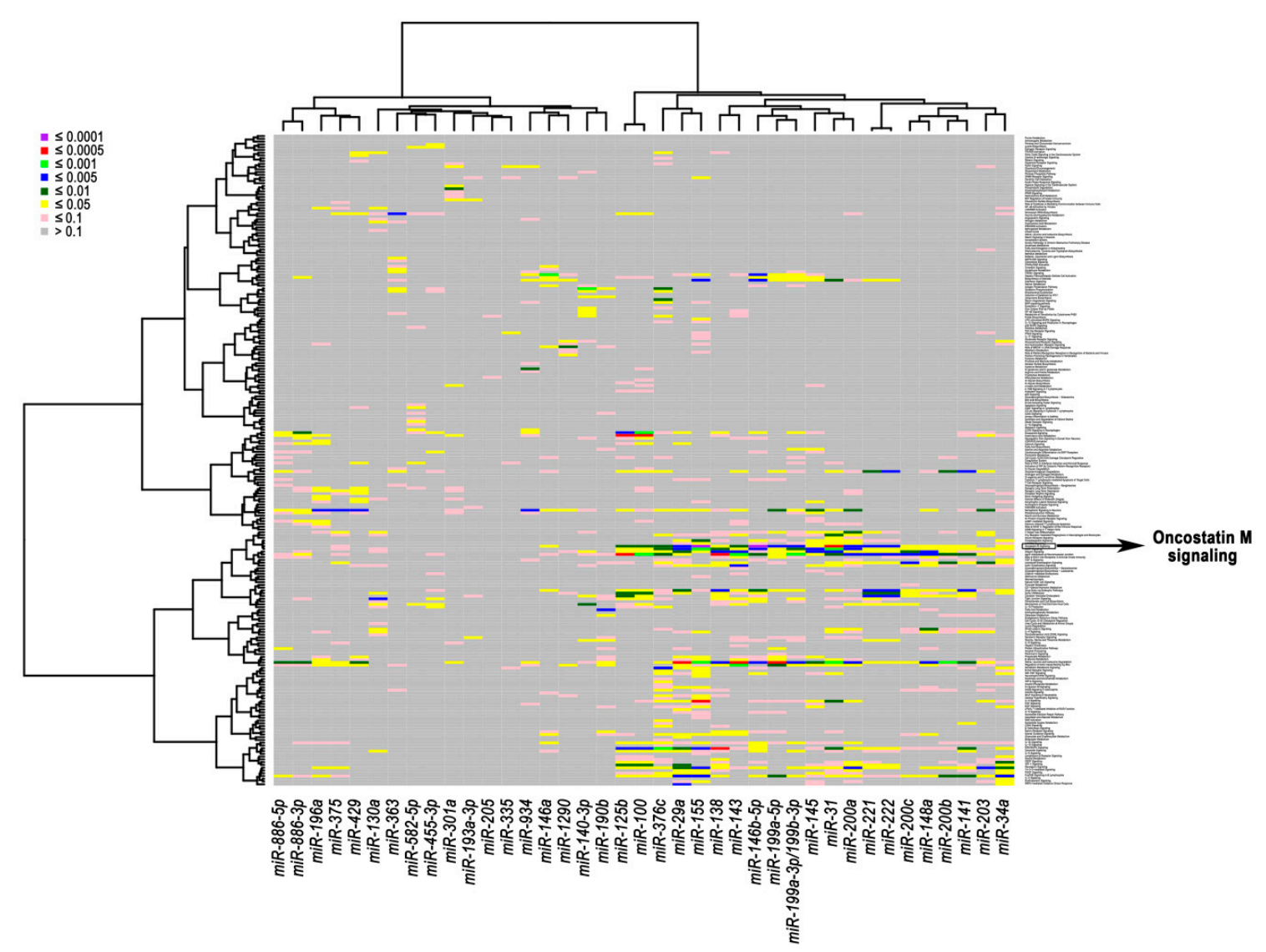

Figure 3. Heatmap of association between correlated miRNAs-mRNAs and signaling pathways in breast cancer cell lines. The heatmap was generated using the expression levels of the 39 selected miRNAs and correlated mRNAs. The miRNA-pathway associations were computed and displayed with eight color levels corresponding to different false discovery rate (FDR) levels. 
Table 2. The most relevant pathways related to miRNA-mRNA networks in breast cancer cell lines.

\begin{tabular}{|c|c|c|}
\hline Signaling Pathway & miRNA & FDR \\
\hline \multirow{5}{*}{ OSM signaling } & miR-146b-5p, miR-155 & $\leq 1.00 \times 10^{-4}$ \\
\hline & miR-31 & $\leq 5.00 \times 10^{-4}$ \\
\hline & miR-29a, miR-199a-3p/199b-3p, miR-199a-5p, miR-200a, miR-221, miR-222 & $\leq 5.00 \times 10^{-3}$ \\
\hline & miR-100, miR-125b, miR-143, miR-376c & $\leq 1.00 \times 10^{-2}$ \\
\hline & $\begin{array}{l}\text { miR-34a, miR-138, miR-141, miR-145, miR-146a, miR-148a, miR-200b, } \\
\text { miR-200c, miR-203 }\end{array}$ & $\leq 5.00 \times 10^{-2}$ \\
\hline \multirow{5}{*}{ ERK/MAPK signaling } & miR-138 & $\leq 5.00 \times 10^{-4}$ \\
\hline & $\operatorname{miR}-376$ & $\leq 1.00 \times 10^{-3}$ \\
\hline & miR-100, miR-125b, miR-155 & $\leq 5.00 \times 10^{-3}$ \\
\hline & miR-29a, miR-31, miR-141 & $\leq 1.00 \times 10^{-2}$ \\
\hline & miR-34a, miR-146b-5p, miR-200a, miR-221, miR-222 & $\leq 5.00 \times 10^{-2}$ \\
\hline JAK/STAT pathway & miR-155 & $\leq 1.00 \times 10^{-2}$ \\
\hline \multirow{2}{*}{ Integrin signaling } & miR-31, miR-138, miR-143, miR-145, miR-148a & $\leq 5.00 \times 10^{-3}$ \\
\hline & miR-29a, miR-200c & $\leq 1.00 \times 10^{-2}$ \\
\hline \multirow{3}{*}{ Interleukin-3 signaling } & miR-155 & $\leq 5.00 \times 10^{-4}$ \\
\hline & miR-31 & $\leq 1.00 \times 10^{-2}$ \\
\hline & miR-29a, miR-145, miR-200a, miR-376 & $\leq 5.00 \times 10^{-2}$ \\
\hline Interleukin -4 signaling & miR-31, miR-128a, miR-141, miR-148a, miR-155, miR-203 & $\leq 5.00 \times 10^{-2}$ \\
\hline Interleukin -6 signaling & miR-140, miR-190b, miR-221, miR-222 & $\leq 5.00 \times 10^{-2}$ \\
\hline \multirow{2}{*}{ IFN- $\gamma$ signaling } & miR-146b, miR-155 & $\leq 5.00 \times 10^{-3}$ \\
\hline & $\operatorname{miR}-31$ & $\leq 1.00 \times 10^{-2}$ \\
\hline EGF signaling & miR-31, miR-145, miR-155 & $<5.00 \times 10^{-2}$ \\
\hline \multirow{2}{*}{ T helper differentiation pathway } & miR-220a & $\leq 1.00 \times 10^{-2}$ \\
\hline & miR-31, miR-130a, miR-145, miR-199a-5p, miR-203, miR-221, miR-375 & $\leq 5.00 \times 10^{-2}$ \\
\hline \multirow{2}{*}{ Semaphorins signaling } & miR-196a, miR-375, miR-429, miR-934 & $\leq 5.00 \times 10^{-3}$ \\
\hline & miR-145, miR-199a-5p, miR-200a, miR-203 & $\leq 1.00 \times 10^{-2}$ \\
\hline \multirow{2}{*}{ Leucocyte extravasation } & $\mathrm{miR}-200 \mathrm{c}$ & $\leq 5.00 \times 10^{-3}$ \\
\hline & miR-200b, miR-203 & $\leq 1.00 \times 10^{-2}$ \\
\hline
\end{tabular}

Abbreviations: EGF, epidermal growth factor; ERK, extracellular signal-regulated kinases; IFN- $\gamma$, interferon- $\gamma$; JAK, janus kinase; MAPK, mitogen-activated protein kinase, OSM, oncostatin M; STAT, signal transducer and activator of transcription.

\subsection{Oncostatin M Expression is Associated with ER-Negative/HER2-Negative Breast Cancer}

Considering the key role of immune responses in breast cancer, we validated our findings by analyzing gene expression data from three publicly available breast cancer datasets, comprising 394 ER-positive (luminal; 56.4\%), 262 ER-negative/HER2-negative (37.5\%), and 43 ER-negative/HER2-positive (6.1\%) breast cancers. We found that the expression of OSM and its receptor $(O S M R)$ were significantly higher in ER-negative/HER2-negative compared with that of luminal/HER2-positive breast cancers (OSM, $p=3.90 \times 10^{-2}$; OSMR, $\left.p=2.80 \times 10^{-3}\right)$ (Figure 4A). We confirmed this finding by analyzing the expression of the OSM protein in luminal $(n=40)$ and triple-negative $(n=40)$ breast cancer samples $\left(p=6.00 \times 10^{-3}\right.$; Figure $\left.4 \mathrm{~B}, \mathrm{C}\right)$.

To identify potential mechanisms mediating the effects of OSM signaling perturbation on tumor cells and the immune microenvironment, we evaluated the correlation between OSM expression and crucial oncogenic pathways, including inflammatory responses, EMT, epidermal growth factor (EGF), mitogen-activated protein kinase (MAPK), and phosphatidylinositol 3-kinase (PI3K) cascades. As expected, in silico analysis revealed that high levels of OSM expression significantly correlated with several genes associated with macrophage functions and immune microenvironment, including ARG1, CCL17, CCL24, CSF2, CXCL11, GAS7, IL1RN, IL4, IL6, and NFKB1 (Table 3). Interestingly, the expression of $O S M$ was also consistently associated with genes involved in EMT, EGF signaling, and downstream pathways, including CDH1, EGFR, MAP2K7, MAP3K2, MTOR, PIK3R2, TGFB1, and ZEB2 (Table 3). Collectively, these results suggest that the deregulation of specific miRNA-mRNA networks 
could support the EMT process and contribute to the modulation of the cancer-immunity crosstalk in ER-negative/HER2-negative breast cancer.

A

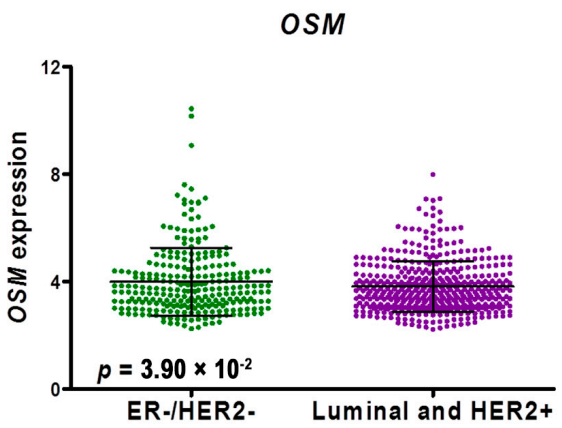

B

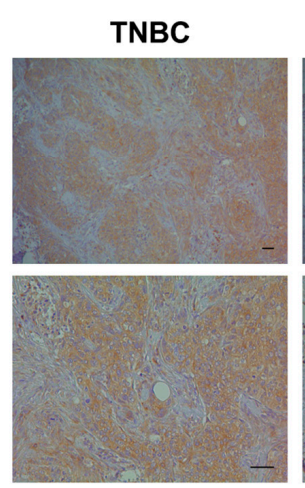

Luminal

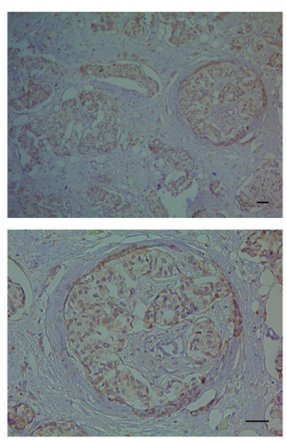

OSMR

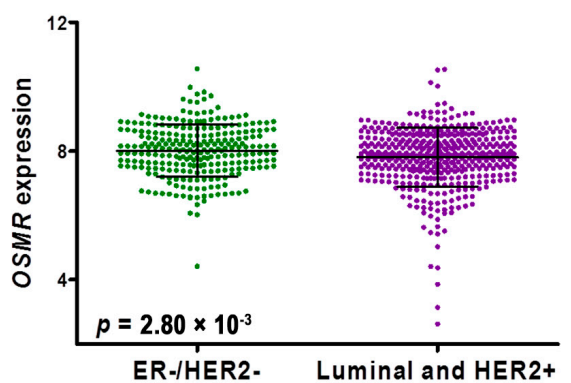

C

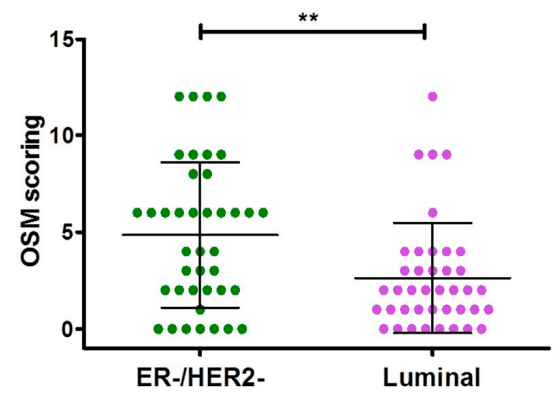

Figure 4. Oncostatin M (OSM) expression levels in different molecular subtypes of breast cancer. (A) Graphs showing OSM and OSM receptor (OSMR) expression levels in estrogen receptor (ER)-negative/human epidermal growth factor receptor 2 (HER2)-negative breast cancer and luminal/HER2-positive subtypes; (B) Representative immunohistochemical images of OSM expression in triple-negative and luminal breast tumors. Scale bars represent $50 \mu \mathrm{m}$; (C) Quantification of staining intensity. Data are presented as mean \pm standard deviation (SD). ${ }^{* *} p<0.01$.

Table 3. Most significant correlations of OSM expression with cancer-related genes in ER-negative/ HER2-negative breast cancers $(n=262)$.

\begin{tabular}{|c|c|c|c|c|}
\hline Category & Gene & $\begin{array}{l}\text { Spearman } \\
\text { Coefficient }\end{array}$ & $95 \%$ CI & $p$-Value \\
\hline \multirow{14}{*}{$\begin{array}{l}\text { Macrophage function } \\
\text { and immune response }\end{array}$} & ALOX15 & 0.442 & $0.34-0.54$ & $<1.00 \times 10^{-4}$ \\
\hline & ARG1 & 0.512 & $0.34-0.54$ & $<1.00 \times 10^{-4}$ \\
\hline & CCL17 & 0.218 & $0.10-0.33$ & $4.00 \times 10^{-4}$ \\
\hline & CCL24 & 0.296 & $0.18-0.41$ & $<1.00 \times 10^{-4}$ \\
\hline & CD40LG & 0.335 & $0.22-0.44$ & $<1.00 \times 10^{-4}$ \\
\hline & CERK & 0.196 & $0.07-0.31$ & $1.50 \times 10^{-3}$ \\
\hline & CHN2 & 0.282 & $0.16-0.39$ & $<1.00 \times 10^{-4}$ \\
\hline & CSF2 & 0.382 & $0.27-0.48$ & $<1.00 \times 10^{-4}$ \\
\hline & CSF3 & 0.451 & $0.35-0.55$ & $<1.00 \times 10^{-4}$ \\
\hline & CSF3R & 0.460 & $0.36-0.55$ & $<1.00 \times 10^{-4}$ \\
\hline & CXCL9 & -0.199 & $-0.32--0.08$ & $1.20 \times 10^{-3}$ \\
\hline & CXCL10 & -0.196 & $-0.31--0.07$ & $1.40 \times 10^{-3}$ \\
\hline & CXCL11 & -0.219 & $-0.33--0.01$ & $3.00 \times 10^{-4}$ \\
\hline & GAS7 & 0.306 & $0.19-0.42$ & $<1.00 \times 10^{-4}$ \\
\hline
\end{tabular}


Table 3. Cont.

\begin{tabular}{|c|c|c|c|c|}
\hline Category & Gene & $\begin{array}{l}\text { Spearman } \\
\text { Coefficient }\end{array}$ & $95 \% \mathrm{CI}$ & $p$-Value \\
\hline \multirow{13}{*}{$\begin{array}{l}\text { Macrophage function } \\
\text { and immune response }\end{array}$} & GBP1 & -0.192 & $-0.31--0.07$ & $1.80 \times 10^{-3}$ \\
\hline & HRH1 & 0.250 & $0.13-0.36$ & $<1.00 \times 10^{-4}$ \\
\hline & $I L 1 R N$ & 0.406 & $0.30-0.51$ & $<1.00 \times 10^{-4}$ \\
\hline & IL4 & 0.378 & $0.27-0.48$ & $<1.00 \times 10^{-4}$ \\
\hline & IL6 & 0.271 & $0.15-0.38$ & $<1.00 \times 10^{-4}$ \\
\hline & IL6R & 0.232 & $0.11-0.5$ & $2.00 \times 10^{-4}$ \\
\hline & IL10 & 0.286 & $0.17-0.40$ & $<1.00 \times 10^{-4}$ \\
\hline & IL15RA & -0.228 & $-0.34--0.11$ & $2.00 \times 10^{-4}$ \\
\hline & IL17A & 0.280 & $0.16-0.39$ & $<1.00 \times 10^{-4}$ \\
\hline & IL32 & -0.162 & $-0.28--0.04$ & $8.80 \times 10^{-3}$ \\
\hline & MERTK & 0.162 & $0.04-0.28$ & $8.60 \times 10^{-3}$ \\
\hline & NFKB1 & 0.385 & $0.27-0.49$ & $<1.00 \times 10^{-4}$ \\
\hline & $T N F$ & 0.376 & $0.26-0.48$ & $<1.00 \times 10^{-4}$ \\
\hline \multirow{16}{*}{$\begin{array}{l}\text { EMT, EGF signaling and } \\
\text { downstream pathways }\end{array}$} & CDH1 & -0.317 & $-0.42--0.20$ & $<1.00 \times 10^{-4}$ \\
\hline & COL1A2 & -0.338 & $-0.44--0.22$ & $<1.00 \times 10^{-4}$ \\
\hline & COL3A1 & -0.310 & $-0.42--0.19$ & $<1.00 \times 10^{-4}$ \\
\hline & $D S P$ & -0.227 & $-0.34--0.10$ & $2.00 \times 10^{-4}$ \\
\hline & EGFR & 0.371 & $0.26-0.47$ & $<1.00 \times 10^{-4}$ \\
\hline & $M A P 2 K 6$ & 0.238 & $0.12-035$ & $1.00 \times 10^{-4}$ \\
\hline & $M A P 2 K 7$ & 0.466 & $0.36-056$ & $<1.00 \times 10^{-4}$ \\
\hline & MAP3K2 & 0.358 & $0.24-0.46$ & $<1.00 \times 10^{-4}$ \\
\hline & МАРК 8 & 0.33 & $0.22-0.44$ & $<1.00 \times 10^{-4}$ \\
\hline & MAPK10 & 0.272 & $0.15-0.38$ & $<1.00 \times 10^{-4}$ \\
\hline & MTOR & 0.385 & $0.27-0.49$ & $<1.00 \times 10^{-4}$ \\
\hline & PIK3R2 & 0.213 & $0.09-0.33$ & $5.00 \times 10^{-4}$ \\
\hline & RHOA & 0.250 & $0.13-0.36$ & $<1.00 \times 10^{-4}$ \\
\hline & $S R C$ & 0.237 & $0.12-0.35$ & $1.00 \times 10^{-4}$ \\
\hline & TGFB1 & 0.446 & $0.34-0.54$ & $<1.00 \times 10^{-4}$ \\
\hline & ZEB2 & 0.160 & $0.04-0.28$ & $9.60 \times 10^{-3}$ \\
\hline
\end{tabular}

Abbreviations: CI, confidence interval; EMT, epithelial-to-mesenchymal transition; EGF, epidermal growth factor.

\section{Discussion}

Numerous studies investigated the value of miRNAs as molecular classifiers, as well as diagnostic, prognostic, and predictive biomarkers in breast cancer [12,17-20]. Since miRNAs are able to modulate multiple oncogenic processes by directly and indirectly regulating gene expression networks, the integration of different -omics data represents a valuable strategy to understand the complex landscape of cancer-related pathways, and to identify novel potential therapeutic targets [21-29]. Accordingly, we showed that miRNA expression profiles are able to accurately characterize molecularly different breast cancer cell lines, demonstrating that a specific pattern of miRNA expression could provide important insights into breast cancer heterogeneity. Noteworthy, the integration of miRNA-mRNA expression profiles allowed for the identification of key deregulated oncogenic pathways, especially immune-related pathways, which could be involved in the development and progression of a specific breast cancer subtype. In particular, we found that the up-regulation of miR-146b and miR-155 in mesenchymal-like TNBC cell lines was consistently associated with perturbation of the OSM pathway, which has been related to reduced expression of ER and poor outcome in breast cancer [30]. Accordingly, our in silico and immunohistochemical analyses demonstrated the significant up-regulation of both the cytokine and its receptor in TNBCs compared with other breast cancer subtypes, supporting a role for the OSM signaling in this more aggressive subgroup of tumors.

Several studies demonstrated the involvement of OSM in the promotion of cell invasiveness and migration, and in the acquisition of mesenchymal-like and stem cell-like features in breast 
cancer [31-34]. Moreover, OSM has been suggested to promote tumor aggressiveness through the modulation of the inflammatory response, especially by inducing the recruitment of macrophages at the tumor site and the polarization toward a pro-tumor M2 phenotype [35-39]. Consistent with the pleiotropic role of OSM signaling in the regulation of mesenchymal-like TNBC cells-immune cells interaction, we found significant associations between OSM levels and crucial oncogenic and immune processes, including macrophage and immune functions, EMT, EGF signaling, and downstream pathways. Therefore, targeting tumor-related inflammation and the cancer-associated immune cells with pro-tumor functions may represent a novel therapeutic approach in mesenchymal-like TNBC.

\section{Materials and Methods}

\subsection{Cell Culture, RNA Isolation, and Microarray Experiments}

The breast cancer cell lines used in this study are described in Table S1. All cell lines were grown and classified according to ATCC, and as previously reported [16]. Total RNA was extracted using the standard TRIzol extraction procedures. After assessing RNA quality and yield with Agilent 2100 Bioanalyzer (Agilent Technologies, Palo Alto, CA, USA) and NanoDrop ND1000 (NanoDrop Technologies, Wilmington, DE, USA), samples were labeled with Cy5/Cy3 using a miRCURY LNA microRNA array Power Labeling kit (Exiqon, Vedbaek, Denmark), and hybridized to Exiqon miRCURY LNA miRNA array V.11.0 (Exiqon), that contains capture probes for all miRNAs registered in the miRBase version 11.0 at the Sanger Institute (available on: http://www.mirbase.org). The quality of labeling reaction and hybridization was evaluated using spike-in controls. Background corrected and $\log 2$ transformed expression data were normalized using the global locally weighted scatterplot smoothing (Lowess) regression algorithm, which we have found to produce the best within-slide normalization and minimize the intensity-dependent differences between the dyes. Gene expression profiling of breast cancer cell lines was performed using U133A Affymetrix chips containing 22,283 probe sets (Affymetrix, Santa Clara, CA, USA). Expression data were processed and normalized in the R environment using the Robust Multiarray Analysis (RMA) algorithm implemented in the Affy Bioconductor package (available on: http:/ / www.bioconductor.org).

\subsection{Human Breast Cancer Datasets and in Silico Analysis}

To evaluate the biological crosstalk between interesting deregulated pathways in breast cancer, publicly available gene expression profiles of breast cancer patients $(n=699)$ were collected from the Gene Expression Omnibus (GEO) database (GSE25066, GSE23988, GSE20194). Gene expression data were generated with Affymetrix U133A gene chips (Affymetrix) and normalized with the Microarray Analysis Suite 5 (MAS5) algorithm, mean centered to 600 and $\log 2$ transformed using the Affy Bioconductor library $[4,5]$. For genes targeted by multiple microarray probes, only the probe set with the highest JetSet score was selected. ER and HER2 status were determined for each patient using the probe sets 205225_at and 216836_s_at, respectively. Normalized values $>10.18$ of the probe set 205225_at were considered as ER-positive, and values > 12.54 for the probe set 216836_s_at were considered as HER2-positive.

\subsection{Tumor Samples and Immunohistochemistry}

Formalin-fixed, paraffin-embedded (FFPE) tissues were retrospectively collected from patients with histologically confirmed invasive ductal luminal $(n=40)$ or triple-negative $(n=40)$ breast cancers, who underwent surgery at Humanitas Clinical and Research Institute (Rozzano-Milan, Italy). FFPE sections $(3 \mu \mathrm{m})$ were incubated with an anti-OSM antibody (Sigma-Aldrich, Milan, Italy) for $1 \mathrm{~h}$ at room temperature. After washing with phosphate buffered saline (PBS), MACH 1 Universal horseradish peroxidase (HRP) Polymer (Biocare Medical, Concord, CA, USA), and diaminobenzidine (DAB; Biocare Medical) were used for chromogenic immunodetection, followed by counterstaining with hematoxylin. Negative control slides without primary antibody and positive controls were used 
for each immunostaining run. Images were captured using an Olympus BX53 microscope (Olympus, Tokyo, Japan). Semi-quantitative analysis of the staining intensity score was performed as previously described [40]. Briefly, the staining intensity was assessed on a scale from 0 to 3 ( 0 , negative; 1 , weak; 2 , moderate; 3 , strong), and staining percentage was scored from 0 to $4(0,0 \%-5 \%$ staining; 4 , $75 \%-100 \%$ staining). A composite score was then calculated by multiplying the staining intensity by the staining percentage.

\subsection{Statistics and Bioinformatics}

Differentially expressed miRNAs, mRNAs, and proteins were identified using an unpaired $t$-test, and $p$-values were adjusted using a Benjamini-Hochberg multi-test correction as indicated in the text. Spearman's rank correlation test was performed to evaluate the correlation between miRNAs and mRNAs. Hierarchical clustering analysis for miRNAs expression was performed using Pearson's distance metric and average linkage using GENE-E software (available on: https:/ / software. broadinstitute.org/GENE-E/index.html). Predicted and validated miRNA targets were identified using miRBase version 21.0 (available on: http:/ / www.mirbase.org/), TargetScan version 6.2 (available on: http://www.targetscan.org), and Tarbase version 7 (available on: http://diana.cslab.ece.ntua. gr/tarbase). Ingenuity pathway analysis software (Qiagen, Redwood City, CA, USA) was used to define signaling pathways regulated by specific miRNAs in breast cancer cell lines. Significant miRNA-pathway correlations were identified by controlling the FDR. All tests were two-sided and the level of statistical significance was set at $p \leq 0.05$. Statistical analyses were performed using GraphPad Prism version 5 (GraphPad Software, La Jolla, CA, USA), and R software version 3.2.2 (available on: http://www.r-project.org).

\section{Conclusions}

Collectively, our data show that specific miRNAs may have an important role as modulators of immune and mesenchymal properties in human breast cancer. The identification of deregulated miRNAs and related pathways may increase our understanding of the oncogenic functions that sustain a specific tumor phenotype and provide a potential opportunity to optimize therapeutic strategies in breast cancer.

Supplementary Materials: Supplementary materials can be found at www.mdpi.com/1422-0067/18/1/194/s1.

Acknowledgments: This study was supported by the Associazione Italiana Ricerca sul Cancro (Grant 6251 to Libero Santarpia), and Fondazione Italiana Ricerca sul Cancro (FIRC fellowship 18328 to Giulia Bottai). The authors want to thank Delphine Boulbes and Bailiang Wang (for technical support with the cell lines).

Author Contributions: Libero Santarpia conceived, designed, and supervised the study; Giulia Bottai, Lixia Diao, and Laura Paladini collected the data; Giulia Bottai, Lixia Diao, and Keith A. Baggerly performed the analysis; Giulia Bottai, Lixia Diao, Lajos Pusztai, Balázs Győrffy, George A. Calin, and Libero Santarpia interpreted the data; Carlotta Raschioni performed immunohistochemistry; Giulia Bottai, Laura Paladini, and Libero Santarpia wrote the manuscript; All authors reviewed and approved the manuscript.

Conflicts of Interest: The authors declare no conflict of interest.

\section{References}

1. Banerji, S.; Cibulskis, K.; Rangel-Escareno, C.; Brown, K.K.; Carter, S.L.; Frederick, A.M.; Lawrence, M.S.; Sivachenko, A.Y.; Sougnez, C.; Zou, L.; et al. Sequence analysis of mutations and translocations across breast cancer subtypes. Nature 2012, 486, 405-409. [CrossRef] [PubMed]

2. Santarpia, L.; Qi, Y.; Stemke-Hale, K.; Wang, B.; Young, E.J; Booser, D.J.; Holmes, F.A.; O'Shaughnessy, J.; Hellerstedt, B.; Pippen, J.; et al. Mutation profiling identifies numerous rare drug targets and distinct mutation patterns in different clinical subtypes of breast cancers. Breast Cancer Res. Treat. 2012, 134, 333-343. [CrossRef] [PubMed]

3. Cancer Genome Atlas Network. Comprehensive molecular portraits of human breast tumours. Nature 2012, $490,61-70$. 
4. Iwamoto, T.; Bianchini, G.; Booser, D.; Qi, Y.; Coutant, C.; Shiang, C.Y.; Santarpia, L.; Matsuoka, J.; Hortobagyi, G.N.; Symmans, W.F.; et al. Gene pathways associated with prognosis and chemotherapy sensitivity in molecular subtypes of breast cancer. J. Natl. Cancer Inst. 2011, 103, 264-272. [CrossRef] [PubMed]

5. Győrffy, B.; Bottai, G.; Fleischer, T.; Munkácsy, G.; Budczies, J.; Paladini, L.; Børresen-Dale, A.L.; Kristensen, V.N.; Santarpia, L. Aberrant DNA methylation impacts gene expression and prognosis in breast cancer subtypes. Int. J. Cancer 2016, 138, 87-97. [CrossRef] [PubMed]

6. Santarpia, L.; Iwamoto, T.; di Leo, A.; Hayashi, N.; Bottai, G.; Stampfer, M.; Andre, F.; Turner, N.C.; Symmans, W.F.; Hortobágyi, G.N.; et al. DNA repair gene patterns as prognostic and predictive factors in molecular breast cancer subtypes. Oncologist 2013, 18, 1063-1073. [CrossRef] [PubMed]

7. Curtis, C.; Shah, S.P.; Chin, S.F.; Turashvili, G.; Rueda, O.M.; Dunning, M.J.; Speed, D.; Lynch, A.G.; Samarajiwa, S.; Yuan, Y.; et al. The genomic and transcriptomic architecture of 2000 breast tumours reveals novel subgroups. Nature 2012, 486, 346-352. [PubMed]

8. Lehmann, B.D.; Bauer, J.A.; Chen, X.; Sanders, M.E.; Chakravarthy, A.B.; Shyr, Y.; Pietenpol, J.A. Identification of human triple-negative breast cancer subtypes and preclinical models for selection of targeted therapies. J. Clin. Investig. 2011, 121, 2750-2767. [CrossRef] [PubMed]

9. Turner, N.C.; Reis-Filho, J.S. Tackling the diversity of triple-negative breast cancer. Clin. Cancer Res. 2013, 19, 6380-6388. [CrossRef] [PubMed]

10. Fridman, W.H.; Pagès, F.; Sautès-Fridman, C.; Galon, J. The immune contexture in human tumours: Impact on clinical outcome. Nat. Rev. Cancer 2012, 12, 298-306. [CrossRef] [PubMed]

11. Savas, P.; Salgado, R.; Denkert, C.; Sotiriou, C.; Darcy, P.K.; Smyth, M.J.; Loi, S. Clinical relevance of host immunity in breast cancer: From TILs to the clinic. Nat. Rev. Clin. Oncol. 2016, 13, 228-241. [CrossRef] [PubMed]

12. Iorio, M.V.; Croce, C.M. MicroRNA dysregulation in cancer: Diagnostics, monitoring and therapeutics. A comprehensive review. EMBO Mol. Med. 2012, 4, 143-159. [CrossRef] [PubMed]

13. Hausser, J.; Zavolan, M. Identification and consequences of miRNA-target interactions-Beyond repression of gene expression. Nat. Rev. Genet. 2014, 15, 599-612. [CrossRef] [PubMed]

14. Kohlhapp, F.J.; Mitra, A.K.; Lengyel, E.; Peter, M.E. MicroRNAs as mediators and communicators between cancer cells and the tumor microenvironment. Oncogene 2015, 34, 5857-5868. [CrossRef] [PubMed]

15. Paladini, L.; Fabris, L.; Bottai, G.; Raschioni, C.; Calin, G.A.; Santarpia, L. Targeting microRNAs as key modulators of tumor immune response. J. Exp. Clin. Cancer Res. 2016, 35, 103. [CrossRef] [PubMed]

16. Neve, R.M.; Chin, K.; Fridlyand, J.; Yeh, J.; Baehner, F.L.; Fevr, T.; Clark, L.; Bayani, N.; Coppe, J.P.; Tong, F.; et al. A collection of breast cancer cell lines for the study of functionally distinct cancer subtypes. Cancer Cell 2006, 10, 515-527. [CrossRef] [PubMed]

17. Iorio, M.V.; Ferracin, M.; Liu, C.G.; Veronese, A.; Spizzo, R.; Sabbioni, S.; Magri, E.; Pedriali, M.; Fabbri, M.; Campiglio, M.; et al. MicroRNA gene expression deregulation in human breast cancer. Cancer Res. 2005, 65, 7065-7070. [CrossRef] [PubMed]

18. Blenkiron, C.; Goldstein, L.D.; Thorne, N.P.; Spiteri, I.; Chin, S.F.; Dunning, M.J.; Barbosa-Morais, N.L.; Teschendorff, A.E.; Green, A.R.; Ellis, I.O.; et al. MicroRNA expression profiling of human breast cancer identifies new markers of tumor subtype. Genome Biol. 2007, 8, R214. [CrossRef] [PubMed]

19. Rothé, F.; Ignatiadis, M.; Chaboteaux, C.; Haibe-Kains, B.; Kheddoumi, N.; Majjaj, S.; Badran, B.; Fayyad-Kazan, H.; Desmedt, C.; Harris, A.L.; et al. Global microRNA expression profiling identifies miR-210 associated with tumor proliferation, invasion and poor clinical outcome in breast cancer. PLoS ONE 2011, 6, e20980. [CrossRef] [PubMed]

20. Volinia, S.; Galasso, M.; Sana, M.E.; Wise, T.F.; Palatini, J.; Huebner, K.; Croce, C.M. Breast cancer signatures for invasiveness and prognosis defined by deep sequencing of microRNA. Proc. Natl. Acad. Sci. USA 2012, 109, 3024-3029. [CrossRef] [PubMed]

21. Michaut, M.; Chin, S.F.; Majewski, I.; Severson, T.M.; Bismeijer, T.; de Koning, L.; Peeters, J.K.; Schouten, P.C.; Rueda, O.M.; Bosma, A.J.; et al. Integration of genomic, transcriptomic and proteomic data identifies two biologically distinct subtypes of invasive lobular breast cancer. Sci. Rep. 2016, 6, 18517. [CrossRef] [PubMed]

22. Hamed, M.; Spaniol, C.; Zapp, A.; Helms, V. Integrative network-based approach identifies key genetic elements in breast invasive carcinoma. BMC Genom. 2015, 16, S2. [CrossRef] [PubMed] 
23. De Rinaldis, E.; Gazinska, P.; Mera, A.; Modrusan, Z.; Fedorowicz, G.M.; Burford, B.; Gillett, C.; Marra, P.; Grigoriadis, A.; Dornan, D.; et al. Integrated genomic analysis of triple-negative breast cancers reveals novel microRNAs associated with clinical and molecular phenotypes and sheds light on the pathways they control. BMC Genom. 2013, 14, 643. [CrossRef] [PubMed]

24. Kristensen, V.N.; Vaske, C.J.; Ursini-Siegel, J.; van Loo, P.; Nordgard, S.H.; Sachidanandam, R.; Sørlie, T.; Wärnberg, F.; Haakensen, V.D.; Helland, Å.; et al. Integrated molecular profiles of invasive breast tumors and ductal carcinoma in situ (DCIS) reveal differential vascular and interleukin signaling. Proc. Natl. Acad. Sci. USA 2012, 109, 2802-2807. [CrossRef] [PubMed]

25. Volinia, S.; Croce, C.M. Prognostic microRNA/mRNA signature from the integrated analysis of patients with invasive breast cancer. Proc. Natl. Acad. Sci. USA 2013, 110, 7413-7417. [CrossRef] [PubMed]

26. Buffa, F.M.; Camps, C.; Winchester, L.; Snell, C.E.; Gee, H.E.; Sheldon, H.; Taylor, M.; Harris, A.L.; Ragoussis, J. MicroRNA-associated progression pathways and potential therapeutic targets identified by integrated mRNA and microRNA expression profiling in breast cancer. Cancer Res. 2011, 71, 5635-5645. [CrossRef] [PubMed]

27. Luo, D.; Wilson, J.M.; Harvel, N.; Liu, J.; Pei, L.; Huang, S.; Hawthorn, L.; Shi, H. A systematic evaluation of miRNA:mRNA interactions involved in the migration and invasion of breast cancer cells. J. Transl. Med. 2013, 11, 57. [CrossRef] [PubMed]

28. Dai, X.; Chen, A.; Bai, Z. Integrative investigation on breast cancer in ER, PR and HER2-defined subgroups using mRNA and miRNA expression profiling. Sci. Rep. 2014, 4, 6566. [CrossRef] [PubMed]

29. Joshi, T.; Elias, D.; Stenvang, J.; Alves, C.L.; Teng, F.; Lyng, M.B.; Lykkesfeldt, A.E.; Brünner, N.; Wang, J.; Gupta, R.; et al. Integrative analysis of miRNA and gene expression reveals regulatory networks in tamoxifen-resistant breast cancer. Oncotarget 2016, 7, 57239-57253. [CrossRef] [PubMed]

30. West, N.R.; Murphy, L.C.; Watson, P.H. Oncostatin M suppresses oestrogen receptor- $\alpha$ expression and is associated with poor outcome in human breast cancer. Endocr. Relat. Cancer 2012, 19, 181-195. [CrossRef] [PubMed]

31. Douglas, A.M.; Grant, S.L.; Goss, G.A.; Clouston, D.R.; Sutherland, R.L.; Begley, C.G. Oncostatin M induces the differentiation of breast cancer cells. Int. J. Cancer 1998, 75, 64-73. [CrossRef]

32. Jorcyk, C.L.; Holzer, R.G.; Ryan, R.E. Oncostatin M induces cell detachment and enhances the metastatic capacity of T-47D human breast carcinoma cells. Cytokine 2006, 33, 323-336. [CrossRef] [PubMed]

33. Guo, L.; Chen, C.; Shi, M.; Wang, F.; Chen, X.; Diao, D.; Hu, M.; Yu, M.; Qian, L.; Guo, N. Stat3-coordinated Lin-28-let-7-HMGA2 and miR-200-ZEB1 circuits initiate and maintain oncostatin M-driven epithelial-mesenchymal transition. Oncogene 2013, 32, 5272-5282. [CrossRef] [PubMed]

34. West, N.R.; Murray, J.I.; Watson, P.H. Oncostatin M promotes phenotypic changes associated with mesenchymal and stem cell-like differentiation in breast cancer. Oncogene 2014, 33, 1485-1494. [CrossRef] [PubMed]

35. Vlaicu, P.; Mertins, P.; Mayr, T.; Widschwendter, P.; Ataseven, B.; Högel, B.; Eiermann, W.; Knyazev, P.; Ullrich, A. Monocytes/macrophages support mammary tumor invasivity by co-secreting lineage-specific EGFR ligands and a STAT3 activator. BMC Cancer 2013, 13, 197. [CrossRef] [PubMed]

36. Tripathi, C.; Tewari, B.N.; Kanchan, R.K.; Baghel, K.S.; Nautiyal, N.; Shrivastava, R.; Kaur, H.; Bhatt, M.L.; Bhadauria, S. Macrophages are recruited to hypoxic tumor areas and acquire a pro-angiogenic M2-polarized phenotype via hypoxic cancer cell derived cytokines Oncostatin M and Eotaxin. Oncotarget 2014, 5, 5350-5368. [CrossRef] [PubMed]

37. Bottai, G.; Raschioni, C.; Székely, B.; di Tommaso, L.; Szász, A.M.; Losurdo, A.; Győrffy, B.; Ács, B.; Torrisi, R.; Karachaliou, N.; et al. AXL-associated tumor inflammation as a poor prognostic signature in chemotherapy-treated triple-negative breast cancer patients. NPJ Breast Cancer 2016, 2, 16033. [CrossRef]

38. Queen, M.M.; Ryan, R.E.; Holzer, R.G.; Keller-Peck, C.R.; Jorcyk, C.L. Breast cancer cells stimulate neutrophils to produce oncostatin M: Potential implications for tumor progression. Cancer Res. 2005, 65, 8896-8904. [CrossRef] [PubMed] 
39. Hollmén, M.; Roudnicky, F.; Karaman, S.; Detmar, M. Characterization of macrophage—Cancer cell crosstalk in estrogen receptor positive and triple-negative breast cancer. Sci. Rep. 2015, 5, 9188. [CrossRef] [PubMed]

40. Zhu, M.; Che, Q.; Liao, Y.; Wang, H.; Wang, J.; Chen, Z.; Wang, F.; Dai, C.; Wan, X. Oncostatin M activates STAT3 to promote endometrial cancer invasion and angiogenesis. Oncol. Rep. 2015, 34, 129-138. [CrossRef] [PubMed]

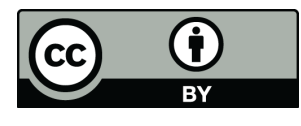

(C) 2017 by the authors; licensee MDPI, Basel, Switzerland. This article is an open access article distributed under the terms and conditions of the Creative Commons Attribution (CC-BY) license (http://creativecommons.org/licenses/by/4.0/). 